\title{
Different Bleeding Patterns with the Use of Levonorgestrel Intrauterine System: Are They Associated with Changes in Uterine Artery Blood Flow?
}

\author{
Carlo Bastianelli, ${ }^{1}$ Manuela Farris, ${ }^{1,2}$ Stefania Rapiti, ${ }^{1,2}$ \\ Roberta Bruno Vecchio, ${ }^{1}$ and Giuseppe Benagiano ${ }^{1}$ \\ ${ }^{1}$ Department of Gynaecology, Obstetrics, and Urology, Sapienza University of Rome, Viale del Policlinico 155, 00161 Rome, Italy \\ ${ }^{2}$ C/O Associazione Italiana Educazione Demografica (AIED), Via Toscana 30, 00187 Rome, Italy
}

Correspondence should be addressed to Manuela Farris; manuela.farris@uniromal.it

Received 17 February 2014; Accepted 26 March 2014; Published 23 April 2014

Academic Editor: Kotaro Kitaya

Copyright ( 2014 Carlo Bastianelli et al. This is an open access article distributed under the Creative Commons Attribution License, which permits unrestricted use, distribution, and reproduction in any medium, provided the original work is properly cited.

\begin{abstract}
Objective. Evaluate if different bleeding patterns associated with the use of the levonorgestrel intrauterine system (LNG-IUS) are associated with different uterine and endometrial vascularization patterns, as evidenced by ultrasound power Doppler analysis. Methodology. A longitudinal study, with each subject acting as its own control was conducted between January 2010 and December 2012. Healthy volunteers with a history of heavy but cyclic and regular menstrual cycles were enrolled in the study. Ultrasonographic examination was performed before and after six months of LNG-IUS placement: uterine volume, endometrial thickness, and subendometrial and myometrial Doppler blood flow patterns have been evaluated. Results. A total of 32 women were enrolled out of 186 initially screened. At six months of follow-up, all subjects showed a reduction in menstrual blood loss; for analysis, they were retrospectively divided into 3 groups: normal cycling women (Group I), amenorrheic women (Group II), and women with prolonged bleedings (Group III). Intergroup analysis documented a statistically significant difference in endometrial thickness among the three groups; in addition, mean pulsatility index (PI) and resistance index (RI) in the spiral arteries were significantly lower in Group I and Group III compared to Group II. This difference persisted also when comparing-within subjects of Group III-mean PI and RI mean values before and after insertion. Conclusions. The LNG-IUS not only altered endometrial thickness, but - in women with prolonged bleedings-also significantly changed uterine artery blood flow. Further studies are needed to confirm these results and enable gynecologists to properly counsel women, improving initial continuation rates.
\end{abstract}

\section{Introduction}

The levonorgestrel-releasing intrauterine system (LNG-IUS) has proven its efficacy, both as a long-acting contraceptive [14] and for its noncontraceptive benefits [5-7], including high effectiveness in the treatment of heavy menstrual bleeding [8-11]. However, prolonged or irregular bleeding during the first months after placement has been reported by $22 \%$ and $67 \%$ of women, respectively, usually declining by the end of the first year [12].

To reduce discontinuations due to the so-called "bleeding nuisances" $[13,14]$, women wishing to use the LNG-IUS should be properly counseled concerning bleeding patterns to be expected [15].

The mechanisms involved in these unexpected patterns remain unclear [8]. A study of endometrial biopsies from LNG-IUS users explored the local effect on the endometrium and documented significant modifications in endometrial vascularization, with a decrease in mean vascular density and an increase in mean vessel area [16]. An increase in subendometrial vascularization has also been documented in women complaining of major side effects (dysmenorrhea and/or irregular bleeding) following insertion of copperreleasing intrauterine devices (Cu-IUDs) [17, 18]. 
A Doppler flow sonographic analysis did not reveal any significant change in uterine artery flow between $\mathrm{Cu}-\mathrm{IUD}$ or LNG-IUS users [19]; a marked reduction in the subendometrial blood flow and endometrial thickness was reported only in the LNG-IUS users. This phenomenon correlates well with morphological changes in the endometrial spiral arteries and capillaries reported in other studies $[20,21]$.

In light of previous work, we have repeated the power Doppler analysis of uterine and endometrial blood flows before and after placement of the LNG-IUS. In addition, to better understand possible influences of the system on uterine and endometrial vascularization, we have attempted to correlate results with various bleeding patterns occurring after insertion.

\section{Methods}

This is a longitudinal study, with each subject acting as its own control; the study was conducted between January 2010 and December 2012 at the Family Planning Centre, Department of Obstetrics, Gynecology and Urology, University La Sapienza, Rome, Italy. The study protocol was approved by the University Ethical Review Committee and was carried out following the principles of the Declaration of Helsinki. All participants provided written, informed consent before entry into the study and after the research protocol was explained in detail verbally.

Healthy women volunteers aged 18-45 years, with a history of regular cycles with heavy menstrual bleedings (cycle length within 21-35 days and menstrual blood loss $>80 \mathrm{~mL}$ per menstruation, as determined by the Higham Pictorial Blood Loss Assessment Chart (PBAC)) and normal cervical smear, were recruited to the study.

Ultrasonographic examination was performed before LNG-IUS placement and six months after insertion with a vaginal probe $5 \mathrm{MH}_{2}$ (Voluson E6 General Electric Ultrasound System), during midluteal phase, 6 to 9 days after ovulation, confirmed by ultrasound. All examinations were carried out by the same investigator, to avoid interobserver variation, and performed between 8.00 and $10.00 \mathrm{am}$.

Women were excluded if they were pregnant (a pregnancy test was performed in all subjects before recruitment) or breastfeeding, if they had a vaginal or caesarean delivery or abortion within 6 weeks of initial screening, and if they had a septic abortion or a postpartum endometritis within 90 days of screening. Subjects were also excluded in the presence of any uterine cavity distortion, past pelvic inflammatory disease, Chlamydia infection, pelvic tuberculosis, or malignancy.

The LNG IUS was then placed within 7 days of the onset of menstruation.

All women were given diary cards to record daily any vaginal bleeding. Bleeding and spotting days and episodes were assessed using the World Health Organization reference period developed by Belsey and Farley [22]. Bleeding intensity was scored according to the PBAC score (a score $>100$ was equivalent to blood loss $>80 \mathrm{~mL}$ ), before entering the study and after 6 months.
The three diameters $(D)$ of the uterus were measured without including the cervix, and uterine volume was then calculated using the formula for an ellipsoid mass $(4 / 3 \times \pi \times$ $D 1 \times D 2 \times D 3)$.

In order to evaluate uterine arteries flow patterns, along with morphological changes in the endometrium in subjects with different bleeding patterns, uterine artery's blood flow velocity waveforms on both sides were evaluated at the level of the inner cervical os; the subendometrial blood flow power Doppler analysis and endometrial thickness measurements were also carried out. The latter was measured as the thickest part in the sagittal section, including both endometrial layers, during the midluteal phase of the menstrual cycle.

Subendometrial blood flow has been assessed utilizing power Doppler energy (PDE) measurement and classified into five categories according to the subendometrial signal area percentage: I (<10\%), II (10-25\%), III (25-50\%), IV (50$75 \%)$, and V (>75\%), as described by others [23].

The Doppler gate was positioned as soon as a vessel with good color signal was obtained and then blood flow velocity waveforms were recorded. The pulsatility (PI: systolediastole) and the resistance (RI: systole-diastole/systole) indices of both uterine arteries were calculated from the mean of three similar consecutive waveforms of good quality. The PI and RI values obtained from each artery were then averaged.

A statistical analysis was performed using SPSS (version 15, Chicago, IL, USA). For quantitative variables, the range, mean, and standard deviation were calculated. The difference between two means was statistically analyzed using the twosided nonpaired $t$-test. The chi-square test was used for categorical data of subendometrial vascularization (power Doppler). A $P$ value of 0.05 was considered statistically significant.

\section{Results}

Out of a total of 186 women screened, 35-all with heavy menstrual bleedings-accepted to participate in the study and 32 were selected after full screening. Two additional subjects were excluded from analysis because of loss to follow-up (Figure 1).

The mean age of women enrolled in the study was 37.3 (range 25-46) years; all had at least a high school degree with a mean PBAC score of $338( \pm 182)$ (Table 1$)$.

Clinically, at six months of follow-up, there was a reduction in menstrual blood loss for all women. Depending on the observed bleeding patterns, for the analysis, participants were divided into 3 groups. Group I included women in whom a cyclical menstrual bleeding pattern was maintained throughout the observation period. Group II comprised women who became amenorrheic after insertion of the system. Group III was made up of women with prolonged or irregular bleedings during treatment.

The three groups were analyzed separately in terms of uterine volume, endometrial thickness, subendometrial blood flow, PI, and RI and no significant association was 


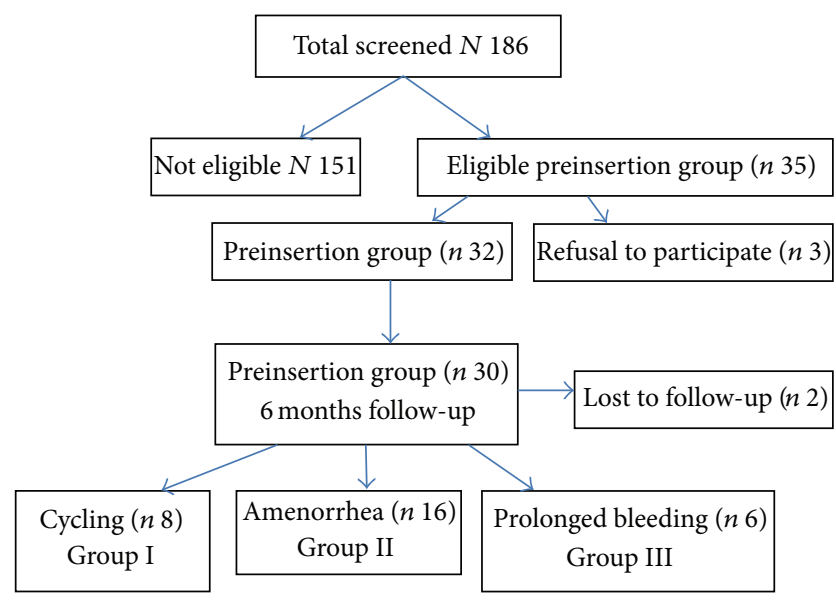

FIGURE 1: Study flow of participating women.

TABLE 1: Women clinical and gynecological characteristics.

\begin{tabular}{|c|c|c|}
\hline & Number & $\%$ \\
\hline \multicolumn{3}{|l|}{ Age } \\
\hline$<30$ & 4 & 12.5 \\
\hline $30-40$ & 16 & 50.0 \\
\hline$>40$ & 12 & 37.5 \\
\hline \multicolumn{3}{|l|}{ Parity } \\
\hline Nulliparous & 4 & 12.5 \\
\hline Parous & 28 & 87.5 \\
\hline \multicolumn{3}{|l|}{$\begin{array}{l}\text { Patterns of menstrual } \\
\text { bleeding }\end{array}$} \\
\hline PBAC SCORE $^{*}$ & $338\left( \pm 182 \mathrm{SD}^{* *}\right)$ & NA \\
\hline \multicolumn{3}{|l|}{$\begin{array}{l}\text { Previous contraceptive } \\
\text { method used }\end{array}$} \\
\hline Oral hormonal & 12 & 37.5 \\
\hline Intrauterine (IUD $\left.{ }^{* * *}\right)$ & 5 & 15.6 \\
\hline Barrier (condom) & 15 & 46.8 \\
\hline
\end{tabular}

found between a woman's obstetrical history, Body Mass Index (BMI), smoking habits, and cycle patterns.

An intergroup analysis disclosed a statistically significant difference in endometrial thickness (Figure 2), while uterine size and subendometrial blood flow did not differ. An additional statistically significant difference was found in endometrial thickness before and after insertion, but only in Group III (Table 2).

The mean PI and mean RI in the spiral arteries were significantly lower in Group I and Group III compared to Group II (Figures 3 and 4). A significant difference was observed also when comparing the mean PI and mean RI values before and after insertion in Group III.

\section{Discussion}

The present study was designed to test the hypothesis that different bleeding patterns observed after placement of

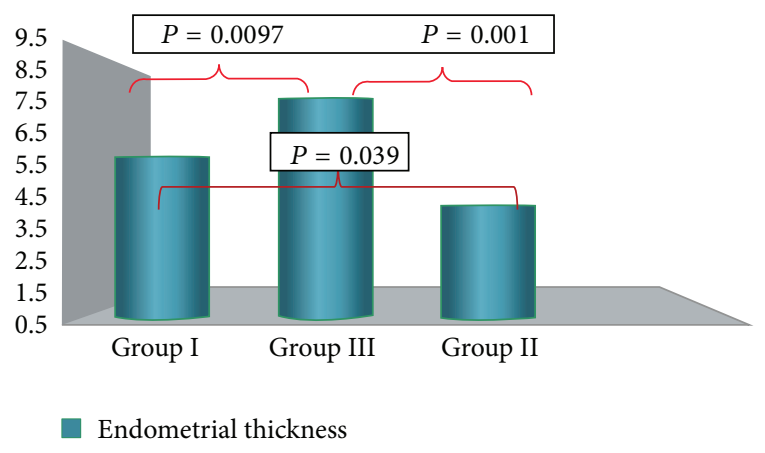

FIGURE 2: Intergroup analysis of endometrial thickness six months after insertion. A significant difference was observed between Group I (normal cycling women), Group II (amenorrheic), and Group III (prolonged bleedings).

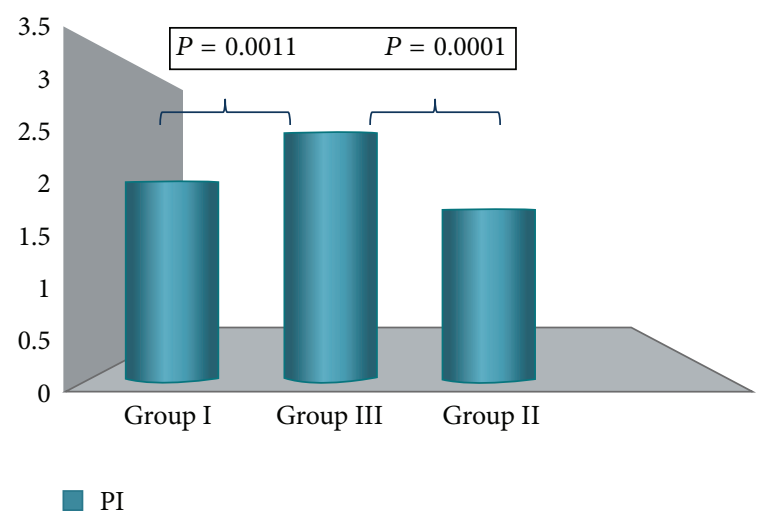

Figure 3: Mean PI values at six months of follow-up (intergroup analysis). A significant difference was observed between Group I (normal cycling women), Group II (amenorrheic), and Group III (prolonged bleedings).

a LNG-IUS may be caused by a different local effect of the system.

Back in 1998, Järvelä et al. [24] conducted a study to measure PI in uterine arteries of women bearing a Cu-IUD and found no significant changes in blood flow after the insertion of the device either during menstruation or in the midluteal phase. At the same time, in women with device-induced dysmenorrhea, a decrease in PI was observed, leading to the conclusion that-overall-the $\mathrm{Cu}$-IUD does not induce any major changes in the resistance of the uterine artery blood flow. In 2006, Jiménez et al. [25] repeated the study and, again, found no significant changes in subendometrial PI and RI, with an endometrial thickness that is lower before than after $\mathrm{Cu}-\mathrm{IUD}$ insertion.

With regard to the effect of the LNG-IUS on uterine arteries Doppler pulsatility patterns results have been contradictory. In 1995, Pakarinen et al. [26] found no changes in PI before and after a 3-month use of the LNG-IUS, in 10 women utilizing the system for contraceptive purposes, while Järvelä et al. [27] concluded that the system increases uterine arteries impedance to blood flow during the midluteal phase, 
TABle 2: Power Doppler analysis, endometrial thickness and uterine volume evaluation at baseline and after 6 months follow up. $P$ refers to intragroup analysis.

\begin{tabular}{lccccccccc}
\hline & \multicolumn{3}{c}{ Group I $(N=8)$} & \multicolumn{2}{c}{ Group II $(N=16)$} & \multicolumn{4}{c}{ Group III $(N=6)$} \\
& Baseline & 6 months & $P$ & Baseline & 6 months & $P$ & Baseline & 6 months & $P$ \\
\hline $\begin{array}{l}\text { Uterine arteries } \\
\quad \text { Mean PI }\end{array}$ & $1.74 \pm 0.49$ & $2.02 \pm 0.47$ & 0.15 & $1.82 \pm 0.62$ & $1.73 \pm 0.27$ & 0.89 & $1.76 \pm 0.52$ & $2.53 \pm 0.33$ & $\mathbf{0 . 0 0 0 1}$ \\
$\begin{array}{l}\text { Uterine arteries } \\
\quad \text { Mean RI }\end{array}$ & $0.79 \pm 0.06$ & $0.82 \pm 0.08$ & 0.27 & $0.78 \pm 0.03$ & $0.83 \pm 0.09$ & 0.17 & $0.77 \pm 0.06$ & $0.92 \pm 0.09$ & $\mathbf{0 . 0 0 0 1}$ \\
$\begin{array}{l}\text { Endom.thickness } \\
\text { Uterine Volume }\end{array}$ & $5.61 \pm 2.89$ & $5.82 \pm 1.77$ & 0.81 & $6.1 \pm 3.21$ & $4.2 \pm 1.48$ & 0.11 & $5.72 \pm 1.78$ & $7.76 \pm 1.17$ & $\mathbf{0 . 0 1}$ \\
\hline
\end{tabular}

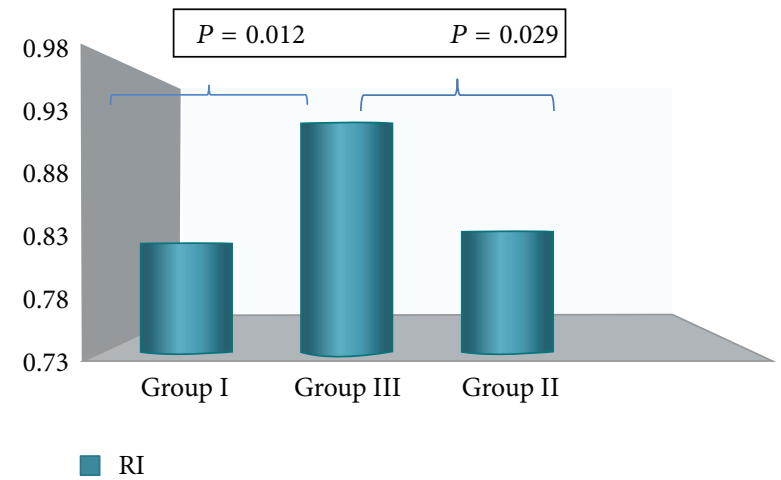

FIGURE 4: Mean RI values at six months of follow-up (intergroup analysis). A significant difference was observed between Group I (normal cycling women), Group II (amenorrheic), and Group III (prolonged bleedings).

a phenomenon that correlates with LNG serum concentrations and a concomitant decrease in serum progesterone levels. The same authors [28] observed in postmenopausal women medicated with transdermal estradiol followed, after one month, by placement of an LGN-IUS a decrease in mean uterine artery PI 1 month after transdermal estradiol treatment; however, the LNG-IUS induced an increase in PI, resulting, at the end of 6 months, in a PI that did not differ significantly from the pretreatment level.

It has been postulated that the progestational effect of the system could induce a change in the subendometrial vascularization. Comparing subendometrial flow in women using the Cu-IUD with those bearing the LNG-IUS, Zalel et al. [29] reported a significant reduction in subendometrial flow in spiral arteries in $75 \%$ of the LNG system users and none in the Cu-IUD-bearing subjects. At the same time, Doppler flow in the cervical branch of the uterine artery did not reveal any changes between the groups. They interpreted these observations as proof of a local progestational effect on the endometrium with no change in the blood flow in the uterine artery. In 2008, Jiménez et al. [30] carried out a similar comparison and found no significant differences in subendometrial vascularization between the groups, with an increase in PI and RI variability (before and after) and a reduction in endometrial thickness in LNG-IUS users. Finally, Haliloglu et al. [31] compared two groups of 60 women, each bearing a Cu- or a LNG-releasing system, before insertion and 1 year after insertion. They observed no change in $\mathrm{Cu}$-IUD users, whereas at one year RI was significantly higher in LNG-IUS users. The PI was also increased at 1 year at a nonsignificant level. Endometrial thickness was also significantly decreased in the postinsertion period in women with LNG-IUS.

In the present study, the LNG-IUS not only altered endometrial thickness, but-in women with prolonged bleedings-also significantly changed uterine artery blood flow. Indeed, mean PI and mean RI of the spiral arteries were significantly lower in normal cycling and in amenorrheic women when compared to women with prolonged bleedings. This difference persisted also when comparing mean PI and mean RI values before and after insertion with the heavy bleeding group.

With regard to uterine arteries' resistance index in women using the LNG-IUS, an older study [29] reported no changes, whereas a more recent one showed an increase [32]. An explanation of these conflicting results may be provided by the present study where no increased RI value was found in amenorrheic women, whereas a significant increase was observed in women with prolonged bleedings.

Mention should be made of the fact that our study design inevitably entailed a selection bias in women with heavy menstrual bleedings. For this reason, we are currently conducting a new trial including also women without HMB.

In conclusion, while we confirmed what was already reported on the effect of the LNG-IUS on endometrial thickness, we also found a significant alteration in uterine artery blood flow, as evidenced by the RI and PI variability, but only in women with prolonged bleedings. This can be a function of a varying local effect of the system.

Further studies are needed to confirm these results in order to better counsel women and reduce discontinuation rates.

\section{Conflict of Interests}

The authors declare that there is no conflict of interests regarding the publication of this paper. 


\section{References}

[1] T. Luukkainen, P. Lahteenmaki, and J. Toivonen, "Levonorgestrel-releasing intrauterine device," Annals of Medicine, vol. 22, no. 2, pp. 85-90, 1990.

[2] T. Luukkainen, “The levonorgestrel-releasing IUD," British Journal of Family Planning, vol. 19, no. 3, pp. 221-224, 1993.

[3] I. Sivin and J. Stern, "Health during prolonged use of levonorgestrel $20 \mu \mathrm{g} / \mathrm{d}$ and the copper TCu 380Ag intrauterine contraceptive devices: a multicenter study," Fertility and Sterility, vol. 61, no. 1, pp. 70-77, 1994.

[4] R. S. French, F. M. Cowan, D. Mansour et al., "Levonorgestrelreleasing $(20 \mu \mathrm{g} /$ day) intrauterine systems (Mirena) compared with other methods of reversible contraceptives," British Journal of Obstetrics and Gynaecology, vol. 107, no. 10, pp. 1218-1225, 2000.

[5] T. Luukkainen, "The levonorgestrel intrauterine system: Therapeutic aspects," Steroids, vol. 65, no. 10-11, pp. 699-702, 2000.

[6] R. Varma, D. Sinha, and J. K. Gupta, "Non-contraceptive uses of levonorgestrel-releasing hormone system (LNG-IUS) - a systematic enquiry and overview," European Journal of Obstetrics Gynecology and Reproductive Biology, vol. 125, no. 1, pp. 9-28, 2006.

[7] J. T. Jensen, "Contraceptive and therapeutic effects of the levonorgestrel intrauterine system: an overview," Obstetrical and Gynecological Survey, vol. 60, no. 9, pp. 604-612, 2005.

[8] J. K. Andersson and G. Rybo, "Levonorgestrel-releasing intrauterine device in the treatment of menorrhagia," British Journal of Obstetrics and Gynaecology, vol. 97, no. 8, pp. 690-699, 1990.

[9] G. W. K. Tang and S. S. T. Lo, "Levonorgestrel intrauterine device in the treatment of menorrhagia in Chinese women: efficacy versus acceptability," Contraception, vol. 51, no. 4, pp. 231235, 1995.

[10] P. Pakarinen and T. Luukkainen, "Treatment of menorrhagia with an LNG-IUS," Contraception, vol. 75, pp. S118-S122, 2007.

[11] H. O. D. Critchley, H. Wang, R. L. Jones et al., "Morphological and functional features of endometrial decidualization following long-term intrauterine levonorgestrel delivery," Human Reproduction, vol. 13, no. 5, pp. 1218-1224, 1998.

[12] J. Suvisaari and P. Lähteenmäki, "Detailed analysis of menstrual bleeding patterns after postmenstrual and postabortal insertion of a copper IUD or a levonorgestrel-releasing intrauterine system," Contraception, vol. 54, no. 4, pp. 201-208, 1996.

[13] T. Sørdal, P. Inki, J. Draeby, M. O’Flynn, and T. Schmelter, "Management of initial bleeding or spotting after Levonorgestrelreleasing intrauterine system placement," Obstetrics \& Gynecology, vol. 121, pp. 934-941, 2013.

[14] M. Rönnerdag and V. Odlind, "Health effects of long-term use of the intrauterine levonorgestrel-releasing system. a followup study over 12 years of continuous use," Acta Obstetricia et Gynecologica Scandinavica, vol. 78, no. 8, pp. 716-721, 1999.

[15] T. Backman, S. Huhtala, R. Luoto, J. Tuominen, I. Rauramo, and M. Koskenvuo, "Advance information improves user satisfaction with the levonorgestrel intrauterine system," Obstetrics and Gynecology, vol. 99, no. 4, pp. 608-613, 2002.

[16] M. Jondet, B. Letellier, and M. T. Verdys, "Endometrial vascularization in levonorgestrel intrauterine device users; computerized microvessel measurement study," Contraception, vol. 71, no. 1, pp. 60-64, 2005.
[17] M. F. Jiménez, D. Vetori, P. A. P. Fagundes, F. M. de Freitas, and J. S. L. Cunha-Filho, "Subendometrial microvascularization and uterine artery blood flow in IUD-induced side effects (levonorgestrel intrauterine system and copper intrauterine device)," Contraception, vol. 78, no. 4, pp. 324-327, 2008.

[18] A. El-Mazny, N. Abou-Salem, and H. Elshenoufy, “Three-dimensional power Doppler study of endometrial and subendometrial microvascularization in women with intrauterine device-induced menorrhagia," Fertility and Sterility, vol. 99, pp. 1912-1915, 2013.

[19] Y. Zalel, A. Shulman, A. Lidor, R. Achiron, S. Mashiach, and R. Gamzu, "The local progestational effect of the levonorgestrelreleasing intrauterine system: a sonographic and Doppler flow study," Human Reproduction, vol. 17, no. 11, pp. 2878-2880, 2002.

[20] P. Zhu, H. Luo, R. Xu et al., "The effect of intrauterine devices, the stainless steel ring, the Copper T220, and releasing levonorgestrel, on the bleeding profile and the morphological structure of the human endometrium - a comparative study of three IUDs," Contraception, vol. 40, no. 4, pp. 425-438, 1989.

[21] R. L. Jones and H. O. D. Critchley, "Morphological and functional changes in human endometrium following intrauterine levonorgestrel delivery," Human Reproduction, vol. 15, no. 3, pp. $162-172,2000$.

[22] E. M. Belsey and T. M. M. Farley, "The analysis of menstrual bleeding patterns: a review," Contraception, vol. 38, no. 2, pp. 129-156, 1988.

[23] M. F. Jiménez, E. P. Passos, P. A. P. Fagundes, J. A. Magalhães, R. Palma-Dias, and J. S. L. Cunha-Filho, "Subendometrial power Doppler quantification: a new classification proposal," Reproductive BioMedicine Online, vol. 11, no. 2, pp. 232-235, 2005.

[24] I. Järvelä, A. Tekay, and P. Jouppila, "The effects of a copper-intrauterine device on the uterine artery blood flow in regularly menstruating women," Human Reproduction, vol. 13, no. 7, pp. 1841-1845, 1998.

[25] M. F. Jiménez, E. P. Passos, P. A. P. Fagundes, F. M. de Freitas, E. Arbo, and J. S. L. Cunha-Filho, "Effect of the copper-intrauterine device (TCu 380A) on subendometrial microvascularization and uterine artery blood flow," Fertility and Sterility, vol. 86, no. 6, pp. 1780-1782, 2006.

[26] P. Pakarinen, T. Luukkainen, H. Laine, and P. Lahteenmaki, "The effect of local intrauterine levonorgestrel administration on endometrial thickness and uterine blood circulation," Human Reproduction, vol. 10, no. 9, pp. 2390-2394, 1995.

[27] I. Järvelä, A. Tekay, and P. Jouppila, “The effect of a levonorgestrel-releasing intrauterine system on uterine artery blood flow, hormone concentrations and ovarian cyst formation in fertile women," Human Reproduction, vol. 13, no. 12, pp. 33793383, 1998.

[28] I. Järvelä, T. Raudaskoski, A. Tekay, and P. Jouppila, "Effect of the levonorgestrel-releasing intrauterine system on the uterine artery pulsatility index in postmenopausal hormone replacement therapy," Ultrasound in Obstetrics and Gynecology, vol. 10, no. 5, pp. 350-355, 1997.

[29] Y. Zalel, R. Gamzu, A. Shulman, R. Achiron, G. Schiff, and A. Lidor, "The progestative effect of the levonorgestrel-releasing intrauterine system-when does it manifest?" Contraception, vol. 67, no. 6, pp. 473-476, 2003. 
[30] M. F. Jiménez, E. Arbo, D. Vetori, F. M. de Freitas, and J. S. L. Cunha-Filho, "The effect of the levonorgestrel-releasing intrauterine system and the copper intrauterine device on subendometrial microvascularization and uterine artery blood flow," Fertility and Sterility, vol. 90, no. 5, pp. 1574-1578, 2008.

[31] B. Haliloglu, A. Celik, E. Ilter, S. Bozkurt, and U. Ozekici, "Comparison of uterine artery blood flow with levonorgestrel intrauterine system and copper intrauterine device," Contraception, vol. 83, no. 6, pp. 578-581, 2011.

[32] A. Haberal, F. Kayikcioglu, M. Gunes, M. Kaplan, and O. Ozdegirmenci, "The effect of the levonorgestrel intrauterine system on uterine artery blood flow 1 year after insertion," Ultrasound in Obstetrics and Gynecology, vol. 27, no. 3, pp. 316-319, 2006. 


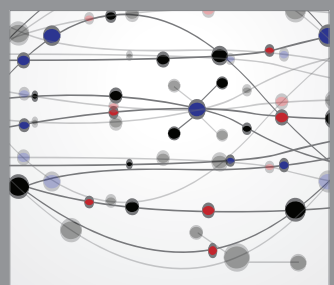

The Scientific World Journal
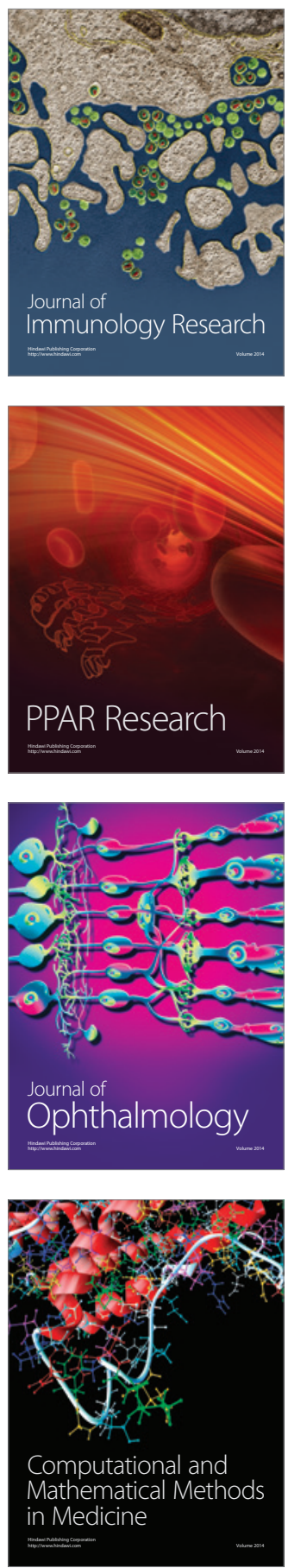

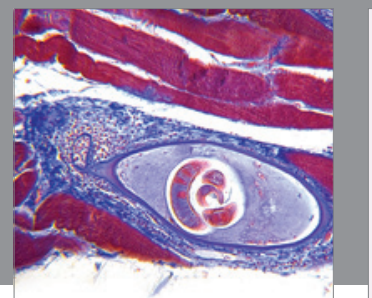

Gastroenterology

Research and Practice
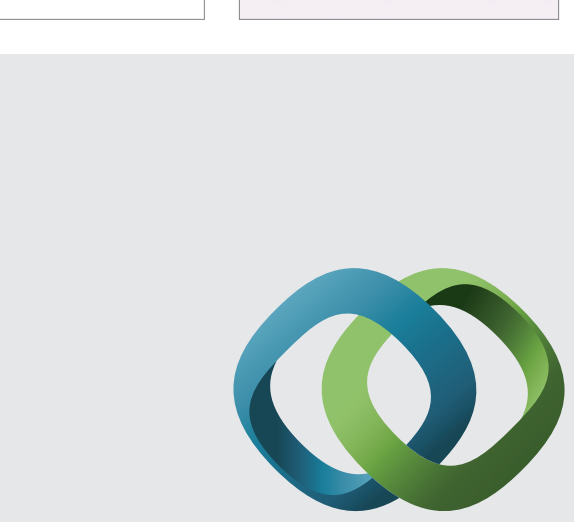

\section{Hindawi}

Submit your manuscripts at

http://www.hindawi.com
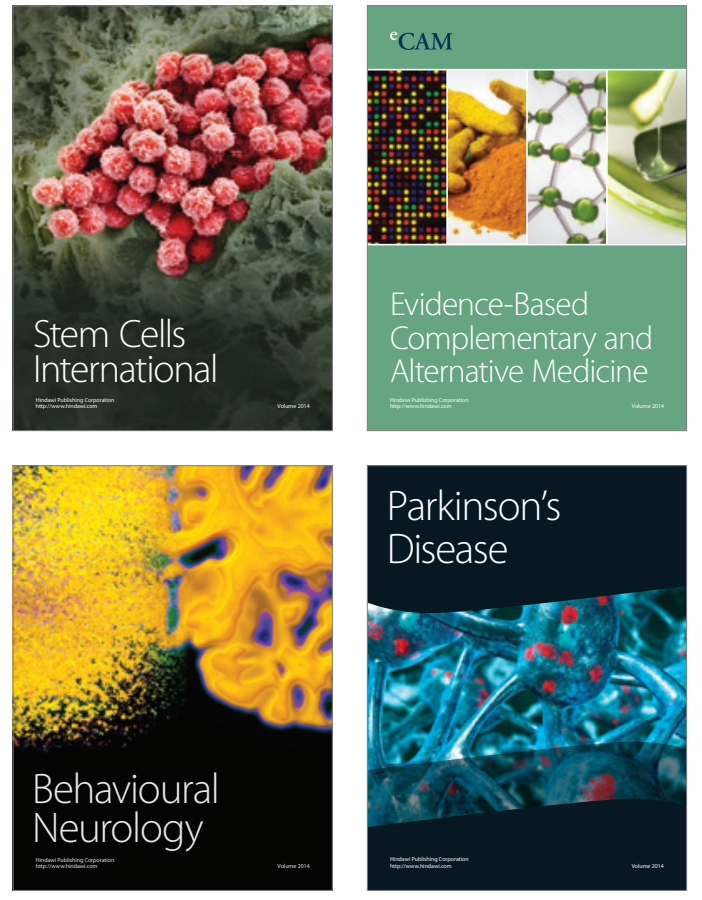
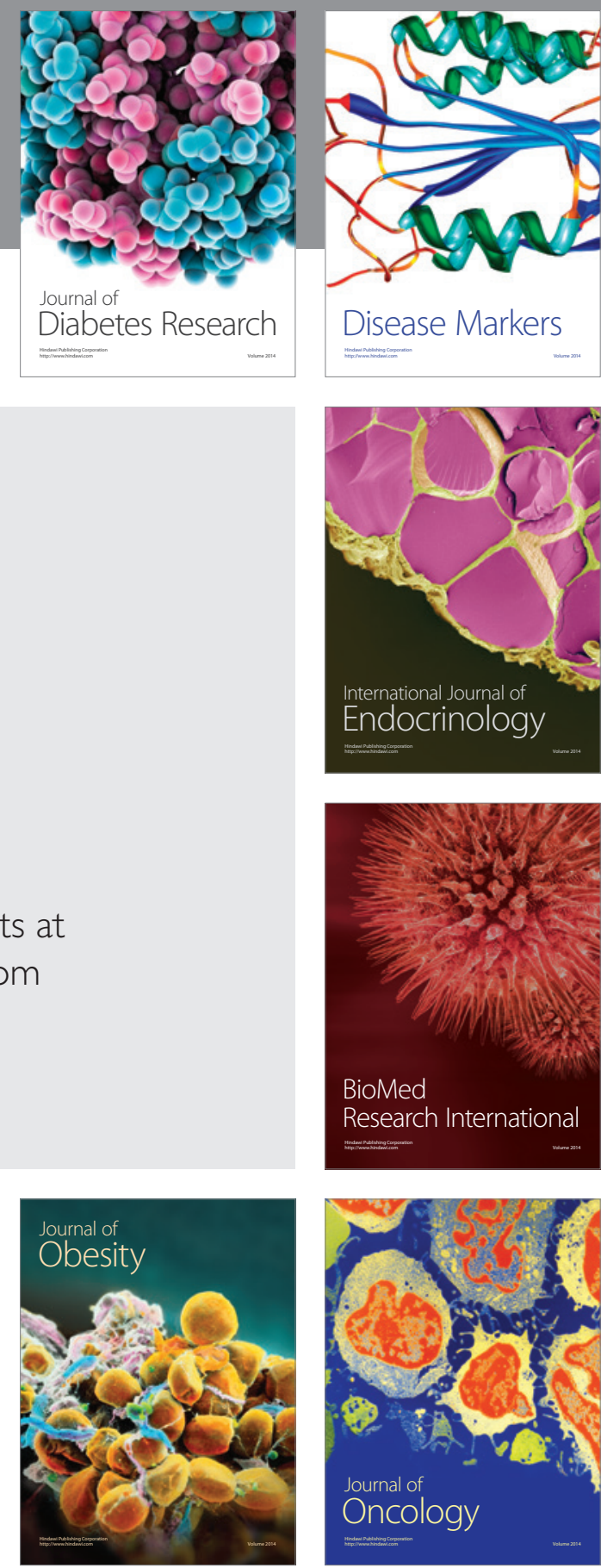

Disease Markers
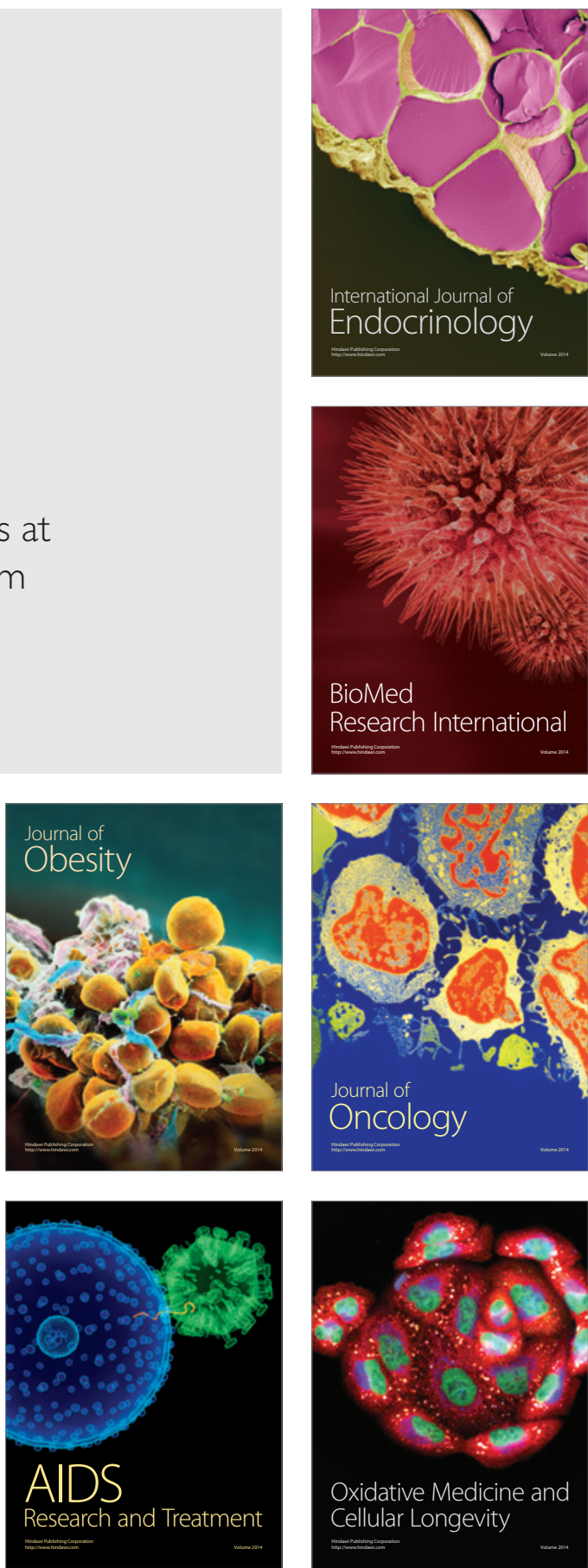